\title{
Spigelian Hernia: Case Series and Review of the Literature
}

\author{
Adeline Rankin Milo Kostusiak Ashraf Sokker \\ Department of General Surgery, The Queen Elizabeth Hospital King's Lynn NHS Trust, King's Lynn, Norfolk, UK
}

\section{Keywords}

Abdominal surgery - Spigelian · Mesh · Hernia . Laparoscopic surgery

\section{Summary}

Background: Spigelian hernia (SH) is an uncommon ventral abdominal hernia. Traditionally repaired with an open technique, the laparoscopic approach is becoming more common and widely described in the literature. We hold that the transabdominal preperitoneal (TAPP) approach restores the anatomy and prevents complications such as seroma. We present a series of $\mathrm{SH}$ repair carried out at a single district general hospital over the past 13 years and a review of the literature. Methods: A retrospective case note review identified $\mathrm{SH}$ repaired between January 2005 and March 2018 at The Queen Elizabeth Hospital, King's Lynn, Norfolk, UK. A Medline search for 'Spigelian hernia' and 'laparoscopic' revealed 41 papers for review. Results: 33 patients underwent repair of SH. We found that the TAPP repair with a mesh is anatomically the most sound repair, with all the added benefits of keyhole surgery, i.e. reduced hospital stay, quicker recovery, and fewer infections. Our complication rates matched those described in the literature. Conclusions: Several operative techniques have been described to repair SH. We favour the laparoscopic TAPP approach which is safe, aids in confirming the correct diagnosis, and has all the benefits of keyhole surgery.

(c) 2018 S. Karger GmbH, Freiburg

\section{Introduction}

The Spigelian or lateral ventral hernia $(\mathrm{SH})$ is a somewhat rare, but probably under-diagnosed abdominal wall hernia. It has variable incidence quoted in the literature ranging from 0.1 to $2 \%$
[1-3]. Named after Adrian van der Spieghel, the 17th century Belgian anatomist who first described the semilunar line, it was not until 1764 that it was first reported as a clinical entity by the Flemish anatomist Josef Klinkosh. SH can be congenital or acquired, appear to peak around the fifth decade, and are slightly more common in women $[1,4]$. Diagnosis of SH can be difficult as they tend to be masked by abdominal fat and the aponeurosis of the external oblique [3]. Surgery is advisable as there is a higher risk of strangulation and incarceration in up to $24 \%$ due to the sharp fascial margin around the defect [5]. Traditionally, SH have been repaired using an open approach with a primary suture repair, or by placing an onlay or sublay mesh. The first case of laparoscopic repair was reported by Carter and Mizes [6] in 1992. Since then, an increasing number of $\mathrm{SH}$ are being repaired laparoscopically because of the advantages of direct visualisation of the defect as well as decreased patient morbidity and hospital stay [7]. The purpose of this study was to present our own case series of $\mathrm{SH}$ repairs and a literature review with the aim of exploring which approaches to laparoscopic repair are both safe and effective.

\section{Patients and Methods}

We carried out a retrospective case note review of patients having undergone a SH repair in our district general hospital between January 2005 and March 2018. Data collected included patient demographics, site of the hernia, whether surgery was elective or emergent, the surgical technique used, and the incidence of postoperative complications and hernia recurrence. Operation notes were reviewed where available and follow-up determined by the last review in a general surgical clinic or by abdominal imaging, e.g. ultrasound or computed tomography (CT). A literature search of the database Medline for the terms 'Spigelian hernia' and 'laparoscopic' was then carried out. Search results were further filtered to include only human adult subjects. 87 articles were retrieved. We discarded articles reporting port-site or incisional Spigelian-type hernia and those without operative details of the laparoscopic repair.

\section{KARGER}

(๑) 2018 S. Karger GmbH, Freiburg 


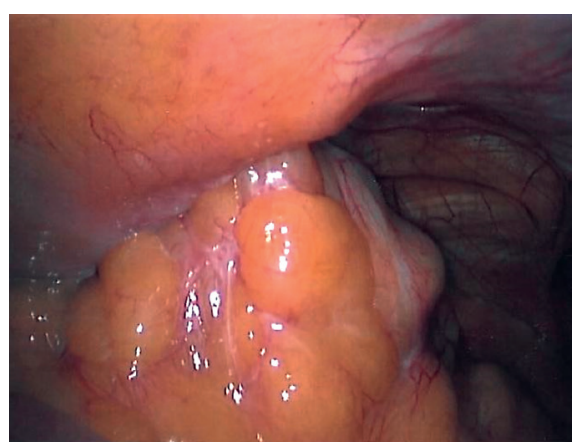

Fig. 1. Spigelian hernia containing omentum.

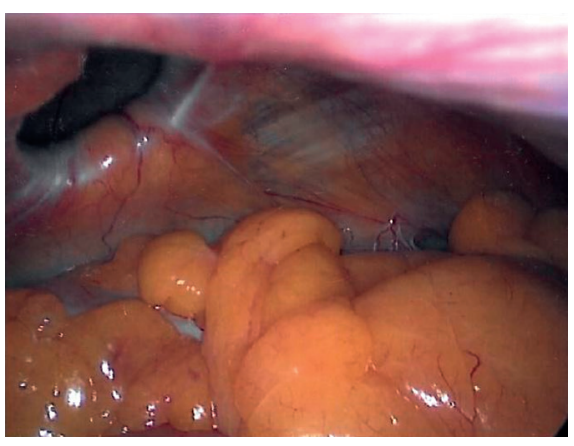

Fig. 2. Abdominal wall defect after reduction of hernia contents.

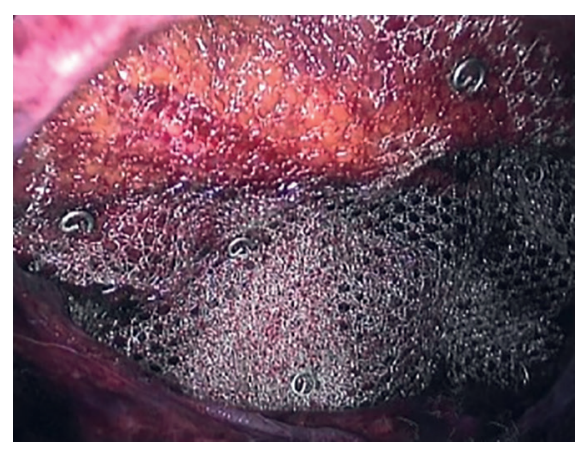

Fig. 3. Preperitoneal mesh.

\section{Results}

\section{Own Series}

Over 13 years, $33 \mathrm{SH}$ repairs were performed. Patient characteristics are displayed in table 1 . The majority of $\mathrm{SH}$ were operated on in an elective setting; the average length of stay was 1.6 days in this group. In those patients requiring emergent repair, the average length of stay was longer at 5.6 days. Imaging was carried out preoperatively on 21 patients (63\%); the majority had CT. Postoperative complications occurred in 7 patients; these included chronic pain requiring referral to chronic pain services, 2 wound infections, 2 patients developed seroma which did not require intervention, 1 patient developed a haematoma which resolved with conservative management and 1 postoperative ileus. The ileus occurred in a patient having a laparoscopic repair. There were no reported recurrences of the hernia, and the mean follow-up was 32 months.

In our laparoscopic repair, we used the transabdominal preperitoneal (TAPP) approach. Patients were positioned supine with the operative surgeon positioned opposite to the hernia site. Pneumoperitoneum is created using the open Hasson technique. A 30-degree laparoscopic camera was introduced via the $11-\mathrm{mm}$ umbilical port. Two $5-\mathrm{mm}$ working ports positioned on the abdominal wall opposite the hernia site create the required triangulation. The defect is located (fig. 1) and the peritoneum surrounding the hernia incised. The sac contents are reduced back into the abdominal cavity (fig. 2); often a preperitoneal crown of fat is present in the defect. The defect is then closed using intracorporal continuous Ethibond 2-0 sutures. A polypropylene mesh is then positioned in the preperitoneal space with at least $5 \mathrm{~cm}$ overlap from the centre of the defect, and fixed with tacks (fig. 3). Peritoneum is then closed over the mesh and the hernia sac everted and fixed to the abdominal wall.

\section{Review of the Literature}

A Medline search of the published literature during the period of 1997-2017 included 87 articles, of which 46 were excluded for reasons described above, leaving 41 articles for review. There were 232 laparoscopic SH repairs (table 2) described in 26 case reports, 10 case series, one prospective trial, and one randomised controlled
Table 1. Patient characteristics of our own case series $(n=33)$

\begin{tabular}{ll}
\hline Male : female & $11: 21$ \\
Mean age, years & 67 (range 51-83) \\
Left side & $14(42 \%)$ \\
Right side & $19(58 \%)$ \\
Emergency surgery & $13(39 \%)$ \\
Elective surgery & $20(61 \%)$ \\
Open repair & $27(82 \%)$ \\
Laparoscopic repair & $6(18 \%)$ \\
Recurrence & none \\
Mean follow-up, months & 32 (range 0-60)
\end{tabular}

trial. Methods of laparoscopic repair included primary suture repair as well as totally extraperitoneal (TEP), intraperitoneal onlay mesh (IPOM), TAPP, and transabdominal partially extraperitoneal repairs. The mean age was 62 years (range $35-87$ ); $37 \%$ were male and $43 \%$ female, with left-sided hernias being slightly more common. The most common method of repair was IPOM (35\%), TEP (30\%), and TAPP (22\%). Of the TAPP repairs only 11 closed the defect prior to mesh placement. Complications included 2 haematomas in the TEP repairs and 3 seromas in the IPOM repairs. Only 2 recurrences were reported: one following primary suture repair and one following IPOM repair.

\section{Discussion}

$\mathrm{SH}$ occur through the Spigelian aponeurosis, defined as the aponeurosis of the transversus abdominus muscle located between the lateral edge of the rectus muscle and the linea semilunaris. Occurring anywhere along the semilunar line, most hernia occur distal to the umbilicus within a belt lying $6 \mathrm{~cm}$ cranial to the interspinal plane $[1,8]$. Spangen [8] describes the hernial sac expanding into the loose space between the internal and external oblique muscles, adopting a typical T- or mushroom-shaped appearance. The diagnosis of $\mathrm{SH}$ can be difficult to make clinically as patients often present with vague abdominal pain with no visible or palpable lump. Imaging with either ultrasound or CT is often required [8]. Surgery is indicated with SH due to the fibrous bands of Spigelian 
Table 2. Literature review of laparoscopic repair of Spigelian hernia

\begin{tabular}{ll}
\hline Cases, $\mathrm{n}$ & 232 \\
Male $:$ female & $88: 108(36 \mathrm{ND})$ \\
Mean age, years & 61 \\
Left $:$ right & $61: 52(131 \mathrm{ND})$ \\
Emergency $:$ elective & $71: 16(146$ mix or ND) \\
Method, $\mathrm{n}=217,12 \mathrm{ND}, 3$ & $\mathrm{PC}=5(2 \%)$ \\
$\quad$ converted to open & $\mathrm{IPOM}=81(36 \%)$ \\
& TAPP $=52(22 \%)$ \\
& TEP $=72(30 \%)$ \\
Defect closed (TAPP), $\mathrm{n}=52$ & TAPE $=7(3 \%)$ \\
Complications, $\mathrm{n}=170(24 \mathrm{ND})$ & $10(20 \%)$ \\
& $2 \times$ haematoma (TEP) \\
& $3 \times$ seroma (IPOM) \\
& ileus (IPOM, TEP) \\
atrial fibrillation (IPOM, TEP) \\
Recurrence, $\mathrm{n}=174$ (58 ND) & $2(1 \%)$
\end{tabular}

$\mathrm{PC}=$ Primary closure; IPOM $=$ intraperitoneal onlay mesh; TAPP $=$ transabdominal preperitoneal; TEP $=$ totally extraperitoneal; TAPE $=$ transabdominal partially extraperitoneal; $\mathrm{ND}=$ not documented.

fascia forming a rigid neck making incarceration and strangulation a risk. The incidence of incarceration has been reported to be around $24-27 \%[5,9]$ and that of strangulation between 2 and $14 \%$ [10]. SH may consist of preperitoneal fat only but may also contain small bowel, colon, or omentum.

The surgical approaches to $\mathrm{SH}$ vary from a traditional open hernia repair using primary sutures or mesh to laparoscopic techniques. In the past 20 years, the laparoscopic approach has become much more common. Laparoscopy has the advantage of offering nearly $100 \%$ diagnostic accuracy [11]. There are two broad approaches to laparoscopic repair of SH, i.e. intraperitoneal or extraperitoneal. The intraperitoneal approach enables the surgeon to examine the peritoneal cavity and abdominal wall to detect coexisting pathology and carry out concomitant procedures. The hernia defect can be repaired by primary suture closure, onlay mesh, or preperitoneal placement of mesh. In younger patients, however, a hernia may only contain extraperitoneal fat with no defect visible from within the peritoneum [12]. In the case series by Webber et al. [3], one third of patients had a SH comprised of extraperitoneal fat only with no peritoneal component; 3 laparoscopic repairs were converted to open because no defect was visible at laparoscopy. The first extraperitoneal approach was published by Moreno-Egea et al. [13] in 1999. It can be carried out under local anaesthetic as an outpatient procedure and avoids the complication of visceral injury associated with the intra-abdominal approach. To date only one prospective randomized trial comparing elective open versus laparoscopic repair of SH has been published [7]. In this study of 22 patients, 11 underwent laparoscopic repair. The intra-abdominal approach was used in 3 patients due to other conditions requiring operative intervention. The remaining hernia were repaired using an extraperitoneal approach. Moreno-Egea et al. [7] found that there was no difference in open or laparoscopic approach when comparing differences in patient characteristics but did find a significant advantage in patient morbidity and length of hospital stay. In a comparison of the extraperitoneal and intra-abdominal techniques, there was no significant difference in morbidity or recurrence rate between the two and the TEP approach was significantly more costly in terms of consumables [9].

The largest case series from Webber et al. [3] describes laparoscopic Spigelian repair in 42 patients. Four different laparoscopic techniques were used, e.g. 19 TEP, 11 IPOM, and 7 TAPE. A hernia staging system was introduced to guide approach strategies. In hernias with no peritoneal component, an open approach is advocated as the defect is not visible from the peritoneum. A moderate-sized defect can be repaired open or laparoscopically, and primary suture repair of small defects is recommended. Larger hernias with significant distortion of the abdominal wall are recommended for open repair due to the possible need for abdominal wall reconstruction. Kelly et al. [14] report another large case series of 40 patients, with 25 having an IPOM repair and the remaining 15 extraperitoneal repair. There were 2 complications in the extraperitoneal group, i.e. haematoma and urinary retention, and 2 in the IPOM group, i.e. atrial fibrillation and ileus. No conclusions were made about the superiority of either approach. With advances in the laparoscopic technique still ongoing, Peterko et al. [15] published a case report of single-incision laparoscopic (SIL) SH repair in 2013. No complications or recurrence were reported; however, the period of follow-up was only 2 months. A case series of $8 \mathrm{SH}$ repairs using the single-incision laparoscopic totally extraperitoneal (SILTEP) technique was published by Tran et al. [16]. One complication of haematoma requiring incision and drainage was reported with no recurrences over a 18-month follow-up.

Barnes et al. [17] published a systematic review investigating the techniques used in the laparoscopic repair of SH. The results were similar to our literature review. Primary suture repair was the least used method. In their case series, Nagarsheth et al. [5] decided not to place a mesh to repair an incidentally found SH during laparoscopic cholecystectomy for acute cholecystitis. This hernia subsequently recurred. IPOM was the most commonly used technique despite the risk of adhesions developing between the peritoneal contents and the mesh $[9,18]$. The defect was not closed in any study. TAPP was the second most used technique, with TEP being the least used. Bittner et al. [19] published case reports describing 2 cases of primary suture closure of small SH defined as $<2 \mathrm{~cm}$ in diameter. At the 12-month follow-up, no complications or recurrence were noted. 10 studies in our literature review describe closure of the fascial defect in larger hernias prior to placement of the mesh in the intraperitoneal approach [20-22]. However, Barnes et al. [18] published a case series of 26 patients in which closure of the fascial defect was purposefully omitted during intraperitoneal repair. No recurrences or complications were seen during a followup period as long as 3.4 years. As previously discussed, these hernia comprise a cap of preperitoneal fat which, if left in situ, we feel may lead to seroma formation and persistence of a lump palpable in the abdominal wall. Yau et al. [23] report inverting the hernia sac and tacking it to prevent seroma formation. 


\section{Conclusion}

In our experience, we conclude that the TAPP approach to SH with reduction of the sac and closure of the defect before placing a preperitoneal mesh reduces the risk of mesh-related complications and seroma formation. SH differs from other ventral hernia in that they do not pass through all layers of the abdominal wall, therefore leaving a cavity and potential space for formation of seroma. Although each laparoscopic approach to $\mathrm{SH}$ has its advantages and disadvantages, it provides a safe and effective repair for the patient. The method employed is dependent on patient characteristics and the surgeon's experience.

\section{Author Contributions}

A.R. designed and carried out the audit and literature review, and wrote the final manuscript. M.K. carried out the audit. A.S. conceived the idea and supervised this work. All authors discussed the results and contributed to the final manuscript.

\section{Ethical Statement}

No ethical approval was required.

\section{Disclosure Statement}

No conflict of interests. No funding was requested or received.

\section{References}

1 Ruiz de la Hermosa A, Amunategui Prats I, Machado Liendo P, Nevarez Noboa F, Muñoz Calero A: Spigelian hernia. Personal experience and review of the literature. Rev Esp Enferm Dig 2010;102:583-586.

2 Takayama S, Imafuji H, Funahashi H, Takeyama H: Laparoscopic Spigelian and inguinal hernia repair with the Kugel patch. Surg Laparosc Endosc Percutan Tech 2010; 20:e76-78.

3 Webber V, Low C, Skipworth RJE, Kumar S, de Beaux AC, Tulloh B: Contemporary thoughts on the management of Spigelian hernia. Hernia 2017;21:355-361.

4 Cinar H, Polat AK, Caglayan K, Ozbalci GS, Topgül HK, Polat C: Spigelian hernia: our experience and review of the literature. Ann Ital Chir 2013;84:649-653.

5 Nagarsheth KH, Nickloes T, Mancini G, Solla JA: Laparoscopic repair of incidentally found Spigelian hernia. JSLS 2011;15:81-85.

6 Carter JE, Mizes C: Laparoscopic diagnosis and repair of Spigelian hernia: report of a case and technique. Am J Obstet Gynecol 1992;167:77-78.

7 Moreno-Egea A, Carrasco L, Girela E, Martín JG, Aguayo JL, Canteras M: Open vs laparoscopic repair of Spigelian hernia: a prospective randomized trial. Arch Surg 2002;137:1266-1268.

8 Spangen L: Spigelian hernia. World J Surg 1989;13: 573-580.
9 Moreno-Egea A, Campillo-Soto Á, Morales-Cuenca G: Which should be the gold standard laparoscopic technique for handling Spigelian hernias? Surg Endosc 2015;29:856-862.

10 Leff DR, Hassell J, Sufi P, Heath D: Emergency and elective laparoscopic repair of Spigelian hernias: two case reports and a review of the literature. Surg Laparosc Endosc Percutan Tec 2009; 19:e152-155.

11 Kasirajan K, Lopez J, Lopez R: Laparoscopic technique in the management of Spigelian hernia. J Laparoendosc Adv Surg Tech A 1997;7:385-388.

12 Williams NS, Bulstrode JK, O'Connell PR: Bailey and Love's Short Practice of Surgery, ed 26. London, CRC Press, 2013.

13 Moreno-Egea A, Torralba JA, Aguayo JL: Totally extraperitoneal laparoscopic repair of Spigelian hernia. Eur J Coeliosurg 1999;32:83-84.

14 Kelly ME, Courtney D, McDermott FD, Heeney A, Maguire D, Geoghegan JG, Winter DC: Laparoscopic Spigelian hernia repair: a series of 40 patients. Surg Laparosc Endosc Percutan Tech 2015;25:e86-89.

15 Peterko AC, Kirac I, Cugura JF, Bekavac-Beslin M: Single incision laparoscopic Spigelian hernia repair an approach with standard instrumentarium. Acta Clin Croat 2013;52:383-386.
16 Tran H, Tran K, Zajkowska M, Lam V, Hawthorne WJ Single-incision laparoscopic repair of Spigelian hernia. ISLS 2015;19:e2015.001644.

17 Barnes TG, McWhinnie DL: Laparoscopic Spigelian hernia repair: a systematic review. Surg Laparosc Endosc Percutan Tech 2016;26:265-270.

18 Barnes TG, McFaul C, Abdelrazeq AS: Laparoscopic transabdominal preperitoneal repair of Spigelian hernia-closure of the fascial defect is not necessary. J Laparoendosc Adv Surg Tech A 2014;24:66-71.

19 Bittner JG 4th, Edwards MA, Shah MB, MacFadyen BV Jr, Mellinger JD: Mesh-free laparoscopic Spigelian hernia repair. Am Surg 2008;74:713-720; discussion 720

20 Palanivelu C, Vijaykumar M, Jani KV, Rajan PS, Maheshkumaar GS, Rajapandian S: Laparoscopic transabdominal preperitoneal repair of Spigelian hernia. JSLS 2006;10:193-198.

21 Patterson A, Thomas B, Franklin A, Connor C, Pullatt R: Transabdominal preperitoneal repair of Spigelian hernia. Am Surg 2016;82:E18-19.

22 Kasirajan K, Lopez J, Lopez R: Laparoscopic technique in the management of Spigelian hernia. J Laparoendosc Adv Surg Tech A 1997;7:385-388.

23 Yau KK, Siu WT, Chan KL, Li KW: A man with recurrent lower abdominal pain: Spigelian hernia. Surg Laparosc Endosc Percutan Tech 2008;18:106-108. 